\title{
Synthesis of a guanidine NHC complex and its application in borylation reactions $\dagger$
}

\author{
Cite this: Chem. Commun., 2014, \\ 50,4344 \\ Received 22nd January 2014, \\ Chia-Cheng Tai, ${ }^{a}$ Ming-Shiuan $\mathrm{Yu}^{\mathrm{b}}{ }^{\mathrm{Y}} \mathrm{Yi-Lin}$ Chen, ${ }^{a}$ Wen-Hang Chuang, ${ }^{a}$ \\ Ting-Hua Lin, ${ }^{a}$ Glenn P. A. Yap ${ }^{c}$ and Tiow-Gan Ong*a
}

Accepted 6th March 2014

DOI: $10.1039 / c 4 c c 00550 c$

www.rsc.org/chemcomm

\begin{abstract}
Synthesis of guanidine-linked NHC can be achieved easily through reacting amino-NHC with carbodiimide. Subsequently, guanidine-NHC $\mathrm{Ag}$ and $\mathrm{Cu}$ complexes were isolated and fully characterized. These $\mathrm{Cu}$ complexes are found to be versatile catalysts for hydroboration, semihydrogenation and carboboration of alkynes in a highly stereoand regioselective fashion.
\end{abstract}

The judicious use of N-heterocyclic carbenes (NHCs) as ligands has resulted in significant advances in the catalysis sector, ${ }^{1}$ attributed to their strong coordination ability and synthetic simplicity with tunable steric and electronic properties. Obviously, covalently attaching an additional functional group on the NHC scaffold would provide additional utilities that may be useful in catalysis. Our group always has ongoing interest in developing catalytic processes based on functional NHC ligands. For example, we have utilized amino-linked NHC to promote Ni-mediated $\mathrm{C}-\mathrm{H}$ bond activation of pyridine and heteroarenes in a selective manner. ${ }^{2}$

In this context, we would like to further extend the architectural diversity and possible catalytic application beyond the realm of amino functional NHC by attaching guanidine to the NHC framework, considering that guanidine has rich chemistry in biological function and organic catalysis. ${ }^{3}$ In this communication, we successfully prepared and characterized guanidine-linked NHC and its copper complex. This guanidine-NHC copper complex has been shown to serve as an efficient and versatile catalyst for hydroboration. Furthermore, we anticipated that vinyl copper intermediate $\mathbf{X}$ may be further exploited for subsequent

\footnotetext{
${ }^{a}$ Institute of Chemistry, Academia Sinica, Taipei, Taiwan, Republic of China. E-mail: tgong@chem.sinica.edu.tw

${ }^{b}$ Department of Chemistry, National Tsing-Hua University, HsiChu, Taiwan, Republic of China

${ }^{c}$ Department of Chemistry \& Biochemistry, University of Delaware, Newark, Delaware, 19716, USA

$\dagger$ Electronic supplementary information (ESI) available: Full experimental and analytical details, including crystallographic data and tables. CCDC 938476 (IPrG-AgBr) and 938477 (IPrG-CuCl). For ESI and crystallographic data in CIF or other electronic format see DOI: 10.1039/c4cc00550c
}

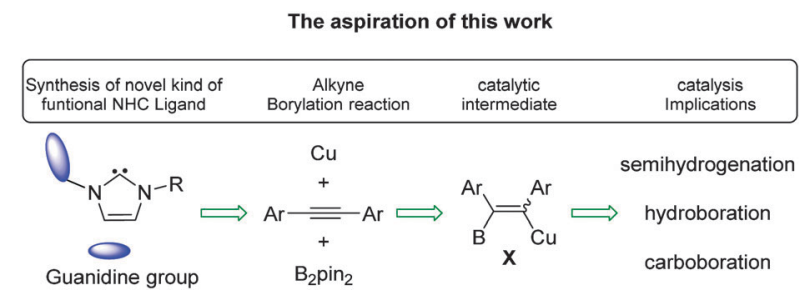

Scheme 1 Strategic plans for developing a versatile copper catalyst based on a guanidine- NHC ligand.

semihydrogenation and carboboration of alkynes using benign synthetic alternatives based on $\mathrm{B}_{2} \mathrm{pin}_{2}$ as shown in Scheme 1 .

As part of our ongoing interest in the development of new catalytic properties based on functional NHC ligands, ${ }^{4}$ we have prepared the first ever imidazolium salt bearing a guanidine pendant arm, IprG, by refluxing carbodiimide with amino-imidazolium salt Me-IM-HBr, followed by treatment with sodium hydroxide as summarized in Scheme 2. Reacting NHC precursor IPrG with $\mathrm{Ag}_{2} \mathrm{O}$ afforded the desired silver NHC transfer adduct IPrG-AgBr. The crystal structure of IPrG-AgBr contains a T-shaped tricoordinated silver bromide complex with two guanidine-NHCs orientated in an anti manner (Scheme 2, left). Subsequently, the copper chloride complex IPrG-CuCl could be readily obtained via transmetallation reaction with IPrG-AgBr. Consistent with

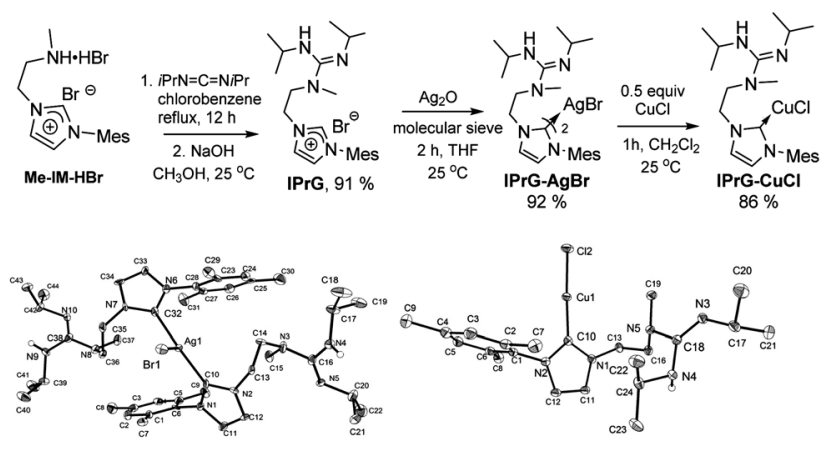

Scheme 2 Synthetic routes and molecular structures of IPrG-AgBr and IPrG-CuCl. 


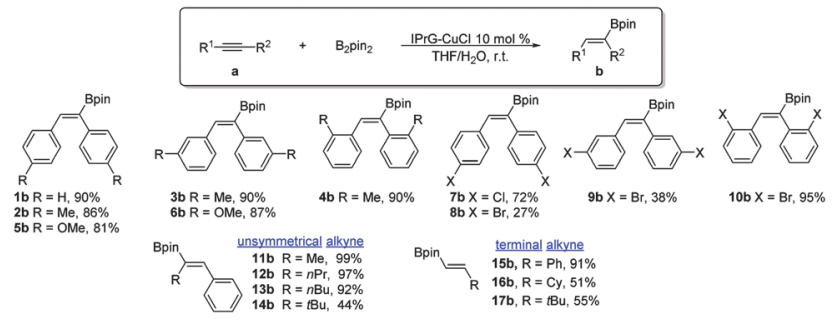

Scheme 3 Hydroboration process with various internal and terminal alkynes. Reaction conditions: a $(0.25 \mathrm{mmol}), \mathrm{B}_{2} \mathrm{pin}_{2}$ (1.3 equiv.), IPrG$\mathrm{CuCl}(10 \mathrm{~mol} \%)$ in THF $(3 \mathrm{~mL})$ and $\mathrm{H}_{2} \mathrm{O}(0.1 \mathrm{~mL})$, room temp for $1 \mathrm{~h}$. Yield of the isolated product.

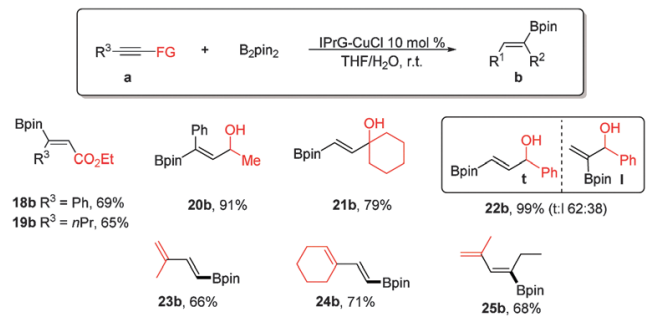

Scheme 4 Hydroboration process with various functional alkynes. Reaction conditions: a $(0.25 \mathrm{mmol}), \mathrm{B}_{2} \mathrm{pin}_{2}$ (1.3 equiv.), IPrG-CuCl (10 mol\%), $\mathrm{NaBr}$ (3 equiv.) in THF $(3 \mathrm{~mL})$ and $\mathrm{H}_{2} \mathrm{O}(0.1 \mathrm{~mL})$ at room temp for $1 \mathrm{~h}$. Yield of the isolated product.

the NMR spectroscopic data and mass analysis, the molecular structure of IPrG-CuCl consists of the copper centre coordinated in a quasi-linear fashion with the NHC and chloride ligands $\left(176^{\circ}\right)$ with a $\mathrm{Cu}-\mathrm{C}$ bond distance of $1.878 \AA$ (Scheme 2, right).

The synthesis of various organoboron compounds via efficient copper-mediated hydroboration of unsaturated substrates would definitely be worthwhile to pursue, particularly the straightforward addition of diboron reagents to carbon-carbon multiple bonds (Schemes 3 and 4). ${ }^{5}$ At this juncture, we have turned our attention to developing the catalytic hydroboration process of alkynes. During the course of optimizing the hydroboration reaction of diphenylacetylene 1a, we found that the combination of catalytic amounts of copper catalyst IPrG-CuCl (10 mol\%), bis(pinacolato)diboron $\left(\mathrm{B}_{2} \mathrm{pin}_{2}, 1.3\right.$ equiv.) and $0.1 \mathrm{~mL}$ of $\mathrm{H}_{2} \mathrm{O}$ ( $\sim 10$ equiv.) in THF would afford a high yield of hydroborated product $1 \mathbf{b}(90 \%)$ with $Z$-stereoselectivity. ${ }^{6}$ With the optimization protocol in hand, we investigated the scope of the reaction with various alkynes. Complete $Z$-selectivity of the hydroborated product and excellent yields were observed for diarylalkyne derivatives bearing electron-donating methyl and methoxy substituents at different positions (2b-6b). High yields were also witnessed for diarylalkynes carrying substituents like $p$-chloro (7b) and $o$-bromo groups (10b), except those with para- and meta-bromo groups, which gave lower yields. The low yield in both reactions is perhaps caused by the strong electronic effect of the bromo substituent at para and meta positions. The unsymmetrically substituted alkyl phenyl alkynes were successfully converted into (Z)-olefinic products 11b-13b. In all cases, borylation occurred perfectly at the $\beta$ carbon to the aryl group with the (Z)-configuration (or geometry). Lastly, terminal alkynes are also suitable substrates in this hydroboration protocol, as exemplified by the formation of 15b-17b products. Unlike other $\mathrm{CuX}$ systems, ${ }^{5}$ no base was required using the IPrG-CuCl catalyst, implying that the dangling guanidine pendant arm might serve as an internal base.

To further expand the utility of this catalytic paradigm, we have examined more challenging alkynes bearing reactive functional groups. Our method exhibits excellent $Z$ - and regioselectivity in the hydroboration of $\alpha, \beta$-unsaturated alkynyl esters to afford products $\mathbf{1 8 b}-\mathbf{1 9 b}$ with the boryl group ended up at the $\beta$ position with respect to the carbethoxy group. The hydroboration of propargyl alcohols is also possible in this method. For example, syn-hydroboration of (20a) and (21a) gave only the $\beta$-regiomer with respect to the hydroxy group in high yields. 1-Phenylprop-2-yn-1-ol (22a) still maintained high regioselectivity (62:38: terminal:internal) with excellent conversion. Finally, we were also delighted to show that such a catalytic hydroboration manifold can be applied to enynes (23a-25a) as well, with average yields of $\sim 60 \%$ without affecting the olefinic functionality of these substrates.

Following the establishment of IPrG-CuCl as a successful hydroboration catalyst, we are curious if hydroboration reactions of unsaturated carbon linkages can be further exploited to mimic the semihydrogenation process to generate olefins in a green manner. A corollary to this hypothesis is that an intermediate such as $\mathbf{X}$ (Scheme 1) might also enable protonation of the $\mathrm{C}-\mathrm{B}$ and $\mathrm{C}-\mathrm{Cu}$ sites by methanol or water under appropriate conditions. Delightfully, the one-step semihydrogenation of diphenylacetylene to $(Z)$-stilbene 1c (99\%) can be achieved in DMF solvent by adding LiOtBu base and maintaining the catalytic conditions similar to those described above for the hydroboration process. With this newly developed protocol in hand, we examined the scope and limitation of the reaction with various diarylalkynes (Scheme 5). Complete $Z$-selectivity of the semihydrogenation products and excellent yields ( 95\%) were observed for diarylalkyne derivatives bearing electron-donating methyl substituents at all positions $\mathbf{2 c - 4 c}$ and methoxy groups at meta (6c). A good yield was witnessed for the reaction of paramethoxy (5c) and para-bromo (8c) while complete selectivity for the $Z$-isomer is still maintained. Gratifyingly, para-chloride (7c) did not seem to perturb the rate or selectivity of the reaction. It is noted that the semihydrogenation transformation can also proceed smoothly with $\mathrm{H}_{2} \mathrm{O}$, acting as an alternative hydrogen source. To the best of our knowledge, this is the first case of selective semi-hydrogenation of alkynes towards (Z)-alkenes with no reduction to the alkanes

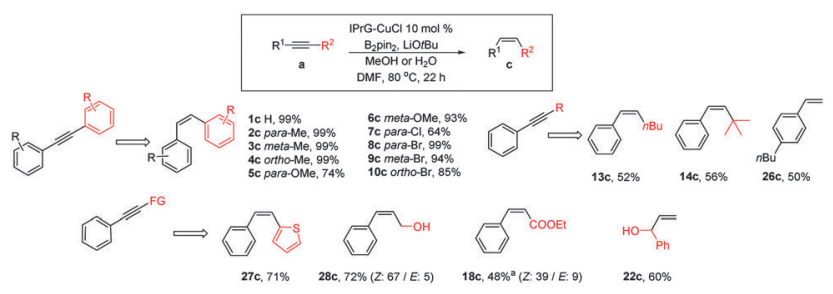

Scheme 5 Semihydrogenation process with various alkynes. Reaction conditions: a (0.28 mmol), $\mathrm{B}_{2}$ pin 2 (1 equiv.), IPrG-CuCl (10 mol\%), LiOtBu (1 equiv.), $\mathrm{MeOH}$ or $\mathrm{H}_{2} \mathrm{O}$ (10 equiv.) in $\mathrm{DMF}(2 \mathrm{~mL}) .{ }^{a} \mathrm{LiBr}$ (3 equiv.). 


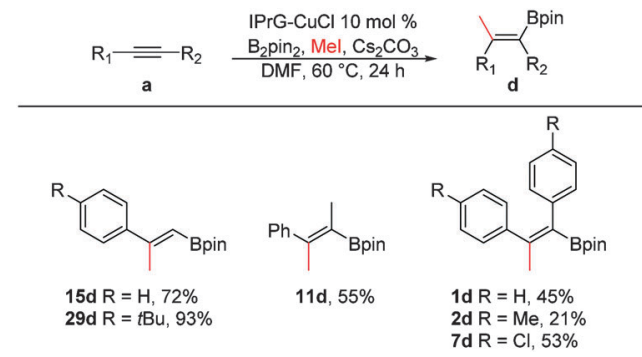

Scheme 6 Carboborylation process with alkynes. Reaction conditions: a $(0.25 \mathrm{mmol}), \mathrm{Mel}\left(4\right.$ equiv.), $\mathrm{B}_{2} \mathrm{Pin}_{2}$ (1.5 equiv.), IPrG-CuCl $(10 \mathrm{~mol} \%)$ $\mathrm{Cs}_{2} \mathrm{CO}_{3}$ (1.5 equiv.) in DMF $(2 \mathrm{ml})$ at $60{ }^{\circ} \mathrm{C}$ for $24 \mathrm{~h}$.

using commercially available boron reagents. Phenylalkynes with alkyl fragments such as linear butyl (13a) or hindering $t$-butyl (14a) as well as terminal alkyne (26a) are also compatible for this catalytic reaction with complete syn stereoseletivity. Similarly, good yield with high $Z$ selectivity was observed for 2-(phenylethynyl)thiophene (27a) and propargyl alcohol derivatives like (28a) and (22a) in semihydrogenation reaction. Equally important, the alkyne derivative bearing the ester moiety (18a) could also be reduced without perturbing the carboethoxy functionality albeit in a lower yield of $48 \%$.

Based on the positive results of the semihydrogenation process, we were curious if a new chemical transformation can further be realized by exploiting the reaction of vinylcopper intermediate $\mathbf{X}$ with other electrophiles like alkyl halide instead of methanol to perform syn-carboboration of simple alkynes. ${ }^{7}$ To our delight, the methylboration of phenylacetylene afforded the desired product $\mathbf{1 5 d}$ in high yield (72\%) in the presence of $\mathrm{B}_{2} \mathrm{pin}_{2}$, methyl iodide and $\mathrm{Cs}_{2} \mathrm{CO}_{3}$ base with catalytic amounts of IPrG-CuCl (Scheme 6). Similarly, internal alkynes like 11a and 1a could be effectively transformed into carboboration adducts 11d and 1d with only one regiomer in a moderate yield, respectively. Other aryl alkyne derivatives like 7a and 29a are also suitable for this reaction, affording a good yield of the final product.

In short, we have successfully prepared and isolated a new kind of functional NHC and its copper complex IPrG-CuCl, namely guanidine NHC. It is found to be a highly active catalyst for hydroboration of various kinds of alkynes bearing active functional groups with a good regioselectivity. More importantly, we have also unraveled a highly efficient catalytic system for the $Z$-selective semihydrogenation of alkynes using commercially available diboron reagents and water or alcohol as viable hydrogen sources, as opposed to the use of hydrogen gas. The versatility of IPrG-CuCl is further illustrated by performing carboboration reaction with alkynes. Further mechanistic investigations of the semihydrogenation process and the development of a more effective catalyst with broader scope are now the focus of our ongoing efforts.

This work was supported by Taiwan National Science Council (NSC: 101-2628-M-001-004-MY3) and Academia Sinica.

\section{Notes and references}

1 (a) W. A. Herrmann, Angew. Chem., Int. Ed., 2002, 41, 1290-1309; (b) F. E. Hahn and M. C. Jahnke, Angew. Chem., Int. Ed., 2008, 47, 3122-3172; (c) S. Díez-González, N. Marion and S. P. Nolan, Chem. Rev., 2009, 109, 3612-3676; (d) D. Enders, O. Niemeier and A. Henseler, Chem. Rev., 2007, 107, 5606-5655.

2 (a) C.-C. Tsai, W.-C. Shih, C.-H. Fang, C.-Y. Li, T.-G. Ong and G. P. A. Yap, J. Am. Chem. Soc., 2010, 132, 11887-11889; (b) W.-C. Shih, W.-C. Chen, Y.-C. Lai, M.-S. Yu, J.-J. Ho, G. P. A. Yap and T.-G. Ong, Org. Lett., 2012, 14, 2046-2049.

3 (a) D. Leow and C.-H. Tan, Chem.-Asian J., 2009, 4, 488; (b) M. P. Coles, Chem. Commun., 2009, 3659.

4 (a) P. L. Arnold, S. A. Mungur, A. J. Blake and C. Wilson, Angew. Chem., Int. Ed., 2003, 42, 5981-5984; (b) P. L. Arnold, M. S. Sanford and S. M. Pearson, J. Am. Chem. Soc., 2009, 131, 13912-13913; (c) H. Jong, B. O. Patrick and M. D. Fryzuk, Can. J. Chem., 2008, 86, 803-810; (d) M. V. Jiménez, J. J. Pérez-Torrente, M. I. Bartolomé, V. Gierz, F. J. Lahoz and L. A. Oro, Organometallics, 2008, 27, 224-234; (e) W. B. Cross, C. G. Daly, R. L. Ackerman, I. R. George and K. Singh, Dalton Trans., 2011, 40, 495-505; ( $f$ ) C. Topf, C. Hirtenlehner, M. Zabel, M. List, M. Fleck and U. Monkowius, Organometallics, 2011, 30, 2755-2764; $(g)$ C. Topf, C. Hirtenlehner and U. Monkowius, J. Organomet. Chem., 2011, 696, 3274-3278; (h) L. Busetto, M. C. Cassani, C. Femoni, M. Mancinelli, A. Mazzanti, R. Mazzoni and G. Solinas, Organometallics, 2011, 30, 5258-5272; (i) B. Ballarin, L. Busetto, M. C. Cassani, C. Femoni, A. M. Ferrari, I. Miletto and G. Caputo, Dalton Trans., 2012, 41, 2445-2455; $(j)$ W. W. N. O, A. J. Lough and R. H. Morris, Organometallics, 2012, 31, 2152-2165; ( $k$ ) Y.-Q. Tang, H. Lv, J.-M. Lu and L.-X. Shao, J. Organomet. Chem., 2011, 696, 2576-2579; $(l)$ F. Nawaz, M. Zaghouani, D. Bonne, O. Chuzel, J. Rodriquez and Y. Coquerel, Eur. J. Org. Chem., 2013, 8253-8264.

5 For recent examples of copper catalyzed borylation reaction, see: (a) H. Yoshida, S. Kawashima, Y. Takemoto, K. Okada, J. Ohshita and K. Takaki, Angew. Chem., Int. Ed., 2012, 51, 235-238; (b) Y. Sasaki, Y. Horita, C. Zhong, M. Sawamura and H. Ito, Angew. Chem., Int. Ed., 2011, 50, 2778-2782; (c) H. R. Kim, I. G. Jung, K. Yoo, K. Jang, E. S. Lee, J. Yun and S. U. Son, Chem. Commun., 2010, 46, 758-760; (d) H. R. Kim and J. Yun, Chem. Commun., 2011, 47, 2943-2945; (e) A. L. Moure, R. Gómez Arrayás, D. J. Cárdenas, I. Alonso and J. C. Carretero, J. Am. Chem. Soc., 2012, 134, 7219-7222; $(f)$ H. Jang, A. R. Zhugralin, Y. Lee and A. H. Hoveyda, J. Am. Chem. Soc., 2011, 133, 7859-7871.

6 See $\mathrm{ESI} \dagger$ for further detail.

7 We were inspired by the recent work on carboboration mediated by $\mathrm{Cu}$ : R. Alfaro, A. Parra, J. Alemań, J. Luis, G. Ruano and M. Tortosa, J. Am. Chem. Soc., 2012, 134, 15165-15168. 